\title{
ANTIMICROBIAL RESISTANCE: UNIVERSAL EMERGENCY
}

\section{Saima Abid ${ }^{1 \otimes}$}

THIS ARTICLE MAY BE CITED AS: Abid S. Antimicrobial resistance: universal emergency. Khyber Med Univ J 2019;II(I):4-5. DOI: 10.35845/kmuj.2019.18987.

A ntimicrobial resistance (AMR) is a growing public health threat. Resistant microorganisms (i.e. bacteria, viruses and some parasites) through genetic mutations become too strong that they become resistant to the antimicrobials (i.e. antibiotics, antivirals and antimalarial) over the period.' These microorganisms are present in our environment, spreading from one person to another and from animals to human beings. ${ }^{2}$

While a considerable burden of AMR is suspected, it is difficult to quantify mortality, disease load and economic loss related to AMR due to the absence of data in Southeast Asia. ${ }^{3}$ AMR causes prolonged illness, surplus tests, consumption of expensive drugs, intensive care, compromised surgeries, chemotherapy, disability and death. AMR can jeopardize the achievements of the Sustainable Development Goals (SDGs).

Resistance is emerging and spreading globally. Many patients with Klebsiella pneumoniae had developed resistance against Carbapenem (last choice for the treatment of gram-negative bacteria) in all regions of the world. Patients with urinary tract infections caused by Escherichia coli are now resistant to fluoroquinolone. In at least 10 developed countries of the world, third generation Cephalosporins are ineffective against the treatment of gonorrhea. World Health Organization (WHO) does not recommend quinolones for the treatment of gonorrhea anymore and has updated treatment guidelines for chlamydial and syphilis infections. Staphylococcus aureus patients are resistant to first-line drug i.e. methicillin. Resistance to colistin, the last remedy for Enterobacteriaceae, has been detected. Alarmingly, extensively drug-resistant tuberculosis (XDR-TB) and multidrug- resistant tuberculosis (MDR-TB) are types of tuberculosis resistant to no less than four and two of the key antituberculous drugs respectively have emerged. XDR-TB has been identified in 105 countries. ${ }^{4}$ About 480,000 new cases of MDR-TB have been estimated by WHO during 2014. In 2016, around 490,000 people developed MDR-TB worldwide. Five countries of the Greater Mekong are now resistant to the artemisinin-based combination therapies considered as first-line treatment for Plasmodium falciparum malaria since 2016. All Anti-Malarial treatment options for Plasmodium falciparum are now ineffective along the Cambodia-Thailand border. About 7\% of patients in developing countries and $10-20 \%$ of patients from developed countries were estimated to have human immunodeficiency virus (HIV) resistant to antiretroviral therapy (ART) in 2010. Antiviral drugs i.e. matrix-2 (M2) inhibitors (Amantadine \& Rimantadine) are now ineffective to treat the Influenza A virus. Outbreaks of multidrug resistance Typhoid and sporadic cases of infection with Ceftriaxone-resistant Salmonella typhi have been reported in several countries of the world.

Recently Pakistan's first extensively drug-resistant (XDR) Salmonella typhi outbreak (resistant to all antibiotics for Typhoid) has created an emergency in the country. The ongoing outbreak had emerged in the district Hyderabad of Pakistan. ${ }^{5}$ In May 2018, the official case definitions were agreed by the Regional Disease Surveillance and Response Unit (RDSRU) in Karachi. Out of the 8, 188 Typhoid cases, 5,274 cases of XDR were reported till December 2018. Among them $69 \%$ of the cases were reported from Karachi, $27 \%$ from Hyderabad and $4 \%$ from other districts of the Sindh province. Currently, Salmonella typhi in circulation is resistant to first, second as
I President Public Health Association, Khyber Pakhtunkhwa \& Associate Professor, Department of Community Medicine and Public Health, Pak International Medical College, Peshawar, Pakistan

Email『 : saimabiddr@gmail.com

well as third generation Cephalosporins. Currently, azithromycin is the only remaining reliable and cost-effective first-line oral therapy to manage patients with XDR Typhoid in low-resource settings.

The risk of XDR Salmonella typhi at the national level is considered high in Pakistan due to insufficient unsafe water supply systems, poor sanitation and hygiene (WASH) practices, poor infection control mechanisms, inappropriate food-handling, low vaccination coverage and limited surveillance of Typhoid fever. At the same time antibiotics are not being prescribed properly to the humans as well as animal sector. Furthermore, AMR confirmatory tests are available only at major laboratories and tertiary care hospitals. All these factors have reduced the ability to track the occurrence, spread and containment of XDR Salmonella typhi in Pakistan.

Access to safe water, adequate sanitation, hospital sanitation and cleanliness, hygiene among food handlers, advocacy, social mobilization, sensitization of general practitioners/ clinicians, Typhoid conjugate vaccination and development of new antibiotics are the most important recommendations for prevention of the AMR concerning XDR Salmonella Typhi in Pakistan..$^{6-7}$ Besides, strengthening the surveillance to monitor known resistance, detect new and emerging trends is the need of the hour..

\section{REFERENCES}

I. World Health Organization (WHO). Antimicrobial Resistance. New WHO Guidelines for the use of medically important antimicrobials in food producing animals. [Cited on: January 20, 2019]. Available from URL: https://www.who.int/ antimicrobial-resistance/en/

2. World Health Organization (WHO). Assessing entry points and options for increasing investments in AMR in 
low- and middle-income countries. [Cited on: January 20, 2019]. Available from URL: https://www.who.int/antimicrobialresistance/national-actionplans/countrycasestudiesinvestmen tsinAMRincountries/en/

3. World Health Organization (WHO). Global Report on Surveillance: Antimicrobial Resistance 2014;I-7. [Cited on: January 20, 2019]. Available from URL: www.who.int/iris/bitstream/10665/ | | 2642/ I/978924| 564748_eng.pdf

4. World Health Organization (WHO). Antimicrobial resistance: Key facts.
[Cited on: January 20, 2019]. Available from URL: http://www.who.int/mediacentre/f actsheets/fs I 94/en.

5. World Health Organization (WHO). Emergencies preparedness, response; Typhoid fever - Islamic Republic of Pakistan; Disease outbreak news; 27 December 2018. [Cited on: January 20, 2019]. Available from URL: https://www.who.int/csr/don/27december-20I8-typhoidpakistan/en/

6. Perez OF, El Chakhtoura NJ, PappWallace KM, Wilson BM, Bonomo
RA. Treatment options for infections caused by carbapenem-resistant Enterobacteriaceae: can we apply "precision medicine" to antimicrobial chemotherapy? Expert Opin Pharmacother 2016; |7:76|-8|. DOI: |0.|5|7/ |4656566.20|6.||45658.

7. Bush K. A resurgence of betalactamase inhibitor combinations effective against multidrug-resistant Gram-negative pathogens. Int J Antimicrob Agents 20 I 5;46:483-93. DOI: $10.1016 / j . i j a n t i m i c a g$. 2015.08.011.
CONFLICT OF INTEREST

Author declared no conflict of interest GRANT SUPPORT AND FINANCIAL DISCLOSURE NIL

\section{(c) (1) (\$) BY NC}

This is an Open Access article distributed under the terms of the Creative Commons Attribution-NonCommercial 2.0 Generic License.
KMUJ web address: www.kmuj.kmu.edu.pk Email address: kmuj@kmu.edu.pk 